\title{
ACTIVE FAULT TOLERANT CONTROL FOR ULTRASONIC PIEZOELECTRIC MOTOR
}

\author{
Moussa Boukhnifer
}

\begin{abstract}
Ultrasonic piezoelectric motor technology is an important system component in integrated mechatronics devices working on extreme operating conditions. Due to these constraints, robustness and performance of the control interfaces should be taken into account in the motor design. In this paper, we apply a new architecture for a fault tolerant control using Youla parameterization for an ultrasonic piezoelectric motor. The distinguished feature of proposed controller architecture is that it shows structurally how the controller design for performance and robustness may be done separately which has the potential to overcome the conflict between performance and robustness in the traditional feedback framework. A fault tolerant control architecture includes two parts: one part for performance and the other part for robustness. The controller design works in such a way that the feedback control system will be solely controlled by the proportional plus double-integral PI2 performance controller for a nominal model without disturbances and $H_{\infty}$ robustification controller will only be activated in the presence of the uncertainties or an external disturbances. The simulation results demonstrate the effectiveness of the proposed fault tolerant control architecture.
\end{abstract}

K e y w or d s: fault tolerant control, robust control, ultrasonic motor

\section{Nomenclature}

\begin{tabular}{ll} 
Symbol & Description \\
\hline$S_{i, j}, D_{n}, \Sigma$ & Strain, Electrical displacement, Entropy \\
$T_{i, j}, E_{n}, \theta$ & Stress, Electrical field, Temperature \\
$s_{i, E, k, l}^{\theta, E}$ & Elastic compliance \\
$\epsilon_{n, m}^{T, \theta}, d_{n, i, j}^{\theta}$ & Electrical permittivity and piezoelectric \\
$p_{m}^{T}, \alpha_{i, j}^{E}$ & $\begin{array}{l}\text { Pyroelectric and thermal expansion } \\
\text { constant }\end{array}$ \\
$\rho \frac{C^{T, E}}{\theta}$ & Thermal constant \\
$(\alpha, \beta),(d, q)$ & Stator's and rotor's reference frames \\
$\underline{v}, \underline{w}, \Psi(\underline{v}, \underline{w})$ & Voltage phasor, Rotating traveling wave, \\
$\theta_{c}, k, R\left(k \theta_{c}\right)$ & Phase \\
$V_{N i d}, V_{T i d}$ & tional matrix \\
$V_{d}, V_{q}$ & Voltal normal and transversal velocity \\
$F_{N}, F_{T}$ & Normal and tangential force \\
$N, T, f_{0}$ & Real rotor speed, Torque, Friction coefficient \\
$f_{r \alpha}, f_{r \beta}$ & Feedback forces in the ( $\alpha, \beta)$ 's reference \\
$A, m$, & frame \\
$d_{s}, c$ & Force factor, Modal mass \\
& Stator's damping term and stiffness
\end{tabular}

\section{INTRODUCTION}

Nowadays, the market for mechatronics systems in such high tech sectors as aeronautics, aerospace, automotive or defense is booming. In these expanding industrial sectors, mechatronics systems are put to test in extreme operating conditions such as thermal cycling or large thermal variations, radiations, corrosion [2], demanding vibratory environments, intense pressures or ultra high vacuum conditions [3], sharp accelerations and severe shocks. As a result the robustness of these embedded mechatronic systems prove to be of paramount importance with respect to their harsh operating environment.

In this context, the ultrasonic motor technology suits mechatronics perfectly due to its powerful performance to compare with its electrical servomotor counterparts. Indeed, its main characteristics are compactness, high torque at low speed without gears, high holding torque without power, low power consumption and fast response. Thus, the ultrasonic motor (USM) has found its place in the industrial sectors. In particular, the traveling wave ultrasonic servomotor (TWUM) has been tested recently by the NASA to operate in cryogenic temperature [4] as well as in ultra high vacuum conditions [3]. Moreover for aeronautics, in the current "fly-by-wire" technology, ultrasonic motor control strategies have been developed to implement active control sticks that allow pilots to feel the force feedback from the rudders $[5,6]$.

Numerous TWUM control strategies have already been implemented in the literature. The speed control strategy [7] with one of the TWUM natural variables (frequency, voltage or phase difference) is the most classical one. However, it is very sensitive to the temperature variations and performances of the motor are not optimized. Another strategy consists in controlling the wave amplitude using the integrated piezoelectric sensor. Though this control is more thermally stable, it is still sensitive to torque variations. Finally, a TWUM model leading to the stationary waves control has also been developed $[6,8,9]$.

* Laboratoire Commande et Systèmes, ESTACA, 34 rue Victor Hugo, F-92300 Levallois-Perret, France, moussa.boukhnifer@estaca.fr 

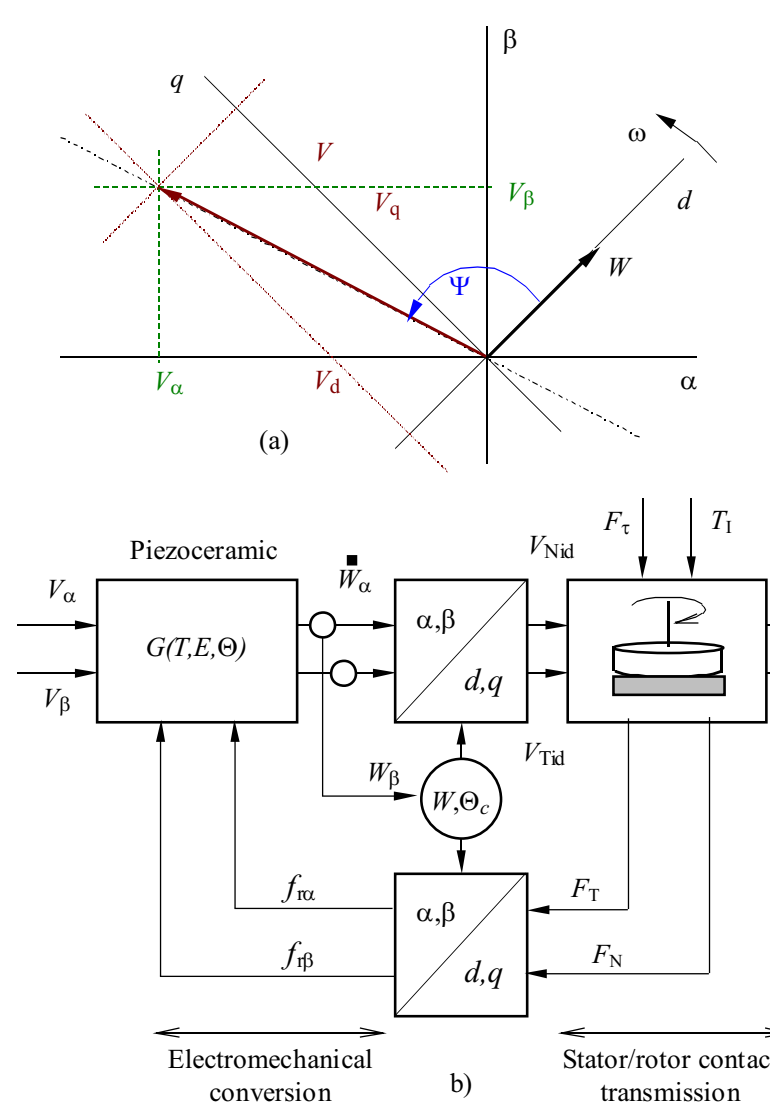

Fig. 1. Causal TWUM model in the stator $(\alpha, \beta)$ reference frame

In [10], the authors have used the model of [5] and used $H_{\infty}$ robust control for the wave and torque loop. In the literature cited thus far, fault tolerance was not considered. However, the faults Sensors affect the systems performance in the closed-loop system when the data sensor is used to define the input. However, even if there is a scheme to detect the fault, due to the nature and confined space of mechatronics, it might not be feasible to intervene and rectify the problem safely in a timely way. Face to the external mechanical disturbances occurring during mechatronics operations a high degree of robustness with good performances with a good fault tolerant control (FTC) is required [1]. It should be mentioned as far as we know, several works have been reported for fault tolerant control of mechatronic systems, ie induction motors [11], hydraulic [12], vehicle [13] driving systems but few works report fault tolerant control of ultrasonic motor. This paper proposes to combine both fault tolerant control and robust control areas to control a piezoelectric ultrasonic motor.

This paper presents a general architecture for fault tolerant control using Youla parametrization for a piezoelectric ultrasonic motor (USM). The distinguished feature of the proposed controller architecture is that it shows structurally how the controller design for performance and robustness may be done separately which has the potential to overcome the conflict between performance and robustness in the traditional feedback framework. When a sen- sor fails or degrades, the controller switches and uses the observer's output instead of the original system's output. The controller architecture includes two parts: the first one for performance and the second one for robustness. The controller architecture works in such a way that the feedback control system will be solely controlled by the proportional plus double-integral PI2 performance controller for a nominal model and the $H_{\infty}$ robust controller will only be activated in the presence of the uncertainties or external disturbances.

This paper can be summarized as follows. An ultrasonic motor model is presented briefly in Section 2. In Section 3, we recall the standard Youla parametrization before introducing the proposed FTC control architecture in Section 4. Then, Section 5 presents the simulation results without and with the proposed robust FTC controller when subjected to external perturbations and faulty sensor operation.

\section{ULTRASONIC MOTOR MODELING}

\subsection{General Layout}

With the objective to implement a robust fault tolerant control strategy for the travelling wave ultrasonic motor, the motor model considered must be neither too simplistic so as to take an accurate account of its nonlinear characteristics, nor overly realistic and consequently hard to implement on a Digital Signal Processor [14] or on a Field Programmable Gate Array [15]. Usually, the ultrasonic motor speed control is implemented and achieved using the equivalent electromechanical model. However, the usual equivalent electrical model though generally sufficient to model the steady-state operation does not accurately model the transient operation. Furthermore, that speed control strategy is very sensitive to thermal and torque variations. Hence, an original causal TWUM model which meets this study objectives was developed recently [5]. Indeed, this original model enables to set up torque control law with a relatively low complexity [5]. The causal TWUM model is described next.

\subsection{Causal TWUM model in the stator's refer- ence frame}

The operation principle of a travelling wave ultrasonic motor is quite similar to that of an induction motor. Indeed, its principle is perfectly similar to the induction motor where the fluctuating magnetic field produced in the air-gap by the two-phase stator supply spawns rotor torque through induction. Consequently, the same mathematical formalism was applied to express the TWUM model through space vectors [10], firstly in the stator's $(\alpha, \beta)$ reference frame, then in the rotating $(d, q)$ frame (as shown in Fig. 1(b)). In a first stage, the two-phase electrical supply, providing respectively the sinusoidal voltages $v_{\alpha}$ and $v_{\beta}$, feeds the two alternate piezoelectric sectors. It results into two purely sinusoidal stationary 
waves, respectively $w_{\alpha}$ and $w_{\beta}$, expressed within the stator's $(\alpha, \beta)$ reference frame. The combination of those vibrating stationary waves propagates consequently along the stator, a rotating travelling wave $\underline{w}$ forming in the $(\alpha, \beta)$ frame an angle $\Psi$ from the voltages phasor $\underline{v}$ (Cf. Figure 1(a)). It is interesting to point out that the TWUM structure provides $k$ permanent contact points with the rotor, corresponding to the $k^{\text {th }}$ excited mode.

Finally, the angular position $\theta_{c}$ and the wave crest $\widehat{w}$ are deduced in the $(\alpha, \beta)$ frame as follows

$$
\begin{aligned}
& \tan \left(k \theta_{c}\right)=\frac{w_{\beta}}{w_{\alpha}}, \\
& \widehat{w}=\sqrt{w_{\alpha}^{2}+w_{\beta}^{2}} .
\end{aligned}
$$

In a second stage, The propagation of the acoustic traveling wave in the stator body makes the individual particles of the stator move in an elliptical fashion. Consequently, the rotor placed on the top of the vibrating stator would be rotated in a direction retrograde to the direction of the traveling wave. That's to say, that the $k$ contacts are considered punctuals with no slidings and no energy storing. This assumption at this point enhances, by means of the rotational matrix $R\left(k \theta_{c}\right)$ in order to express within the $(d, q)$ rotating frame the ideal transversal velocity $V_{\text {Tid }}$ along the quadratic axis $q$ and the ideal normal velocity $V_{N i d}$ along the direct axis $d$. Unfortunately, due to the elastic layer required to improve the contact transmission, the $k$ contacts are not ideal. Actually, the area at the stator/rotor is distributed and a sliding effect occurs, which is essential to provide torque similar to that in induction motor. Numerous previous works have explored modeling of this highly nonlinear contact transmission, resulting in sophisticated models with excellent accuracy. However, in order to implement a straightforward model, despite the fact that the friction coefficient $f_{0}$ varies due to the nonlinear contact transmission, the relation between the real rotor speed $N$ and the torque $T$ is approximated and considered in the overall model as linear

$$
T=f_{0}\left(\frac{1}{b} V_{T_{i d}}-N\right) \quad|T|<T_{\max } .
$$

In addition to the friction phenomenon, the TWUM requires to produce the torque $T$ and consequently to drive the load, a normal force $F_{N}$ to maintain contact condition at the rotor/stator interface as well as a tangential force $F_{T}$. The application of those mechanical stresses results in some feedback forces on the stator. Those feedback forces, respectively $f_{r \alpha}$ and $f_{r \beta}$, are then rotated from the rotating $(d, q)$ frame to the stator's $(\alpha, \beta)$ frame using the previously used rotational matrix $R\left(k \theta_{c}\right)$. At this point, it is important to notice that those feedback forces have a direct influence on the TWUM. Indeed, those external mechanical stresses provoke on the stator side, the piezoelectric ceramic energy to evolve and as a result the resonance pulsation $\omega_{r}$ to drift; which is expressed in the causal TWUM model by $k \theta_{c}$ variations.

\subsection{Model equations in the rotating $(d, q)$ refer- ence frame}

The causal TWUM model expressed in the stator $(\alpha, \beta)$ reference frame enable to implement a straightforward control by means of simplistic mechanical contacts. Nevertheless, it appears that the variable from the normal $\alpha$ axis and the tangential $\beta$ axis are coupled, which is shown through the rotational matrix $R\left(k \theta_{c}\right)$. Thus, so as to remedy to this coupling, the control is then given in the rotating reference frame. The feeding voltages $v_{\alpha}$ and $v_{\beta}$ are then deduced from $V_{d}$ and $V_{q}$ by means of the rotational matrix $R\left(k \theta_{c}\right)$ and the ultrasonic motor equations are deduced for small pulsation variations [5]

$$
\begin{gathered}
m \dot{V}_{N i d}+d_{s} V_{N i d}+\left(c-\left(k \dot{\theta}_{c}\right)^{2}\right) \int V_{N i d} d t=N V_{d}-F_{N}, \\
2 m \dot{V}_{T i d}+d_{s} V_{T i d}=N V_{q}-F_{T}
\end{gathered}
$$

In view of those equations it is interesting to notice that, due to reference frame change, the variables are not coupled. Finally, those model's equations allow to determine the TWUM behaviour in the steady-state and in the transient-state. This straightforward model will be used in the next section in order to implement the FTC robust control.

Figure 2 shows the simulation results of the TWUM behavior with a load torque $T_{L}=0.5 \mathrm{Nm}$ at $50 \mathrm{msec}$.

\section{FTC CONTROLLER ARCHITECTURE}

In this section, Youla parametrization is used in a different way and the use the FTC architecture in order to ensure both performance and robustness outcomes is explained.

Firstly, we consider the feedback diagram presented in Fig. 3, in which the reference signal $r$ enters into the system from a different location. Nevertheless, the internal stability of the system is not changed since the transfer function from $y$ to $u$ is not changed. Thus, this controller implementation also stabilizes internally the feedback system with plant $P_{0}$ for any $Q \in H_{\infty}$ such that $\operatorname{det}(\tilde{V}(\infty)-Q(\infty) \tilde{N}(\infty)) \neq 0$.

Due to the similarity with the well-known IMC (Internal Model Control), we shall call proposed controller framework as generalized internal model control (GIMC) [16].

The distinguished feature of this controller implementation is that the inner loop feedback signal $f$ is always zero, ie $f=0$, if the plant model is perfect, ie if $G=G_{0}$. The inner loop is only active when there is a model uncertainty or other sources of uncertainties such as disturbances and sensor noises [17]. Thus, $Q$ can be designed to robustify the feedback system. It follows that the new controller design architecture has a clear separation between performance and robustness [18]. 

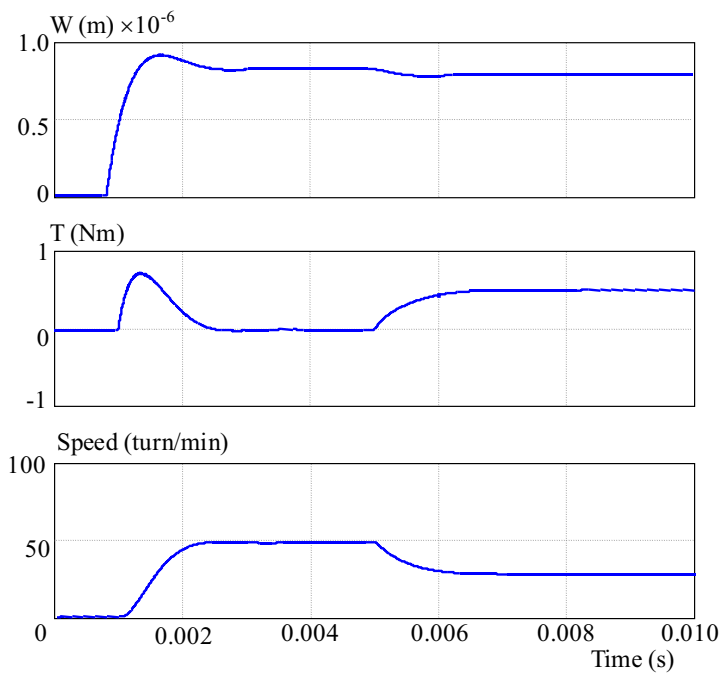

Fig. 2. Simulation results of the causal $T W U M$ model

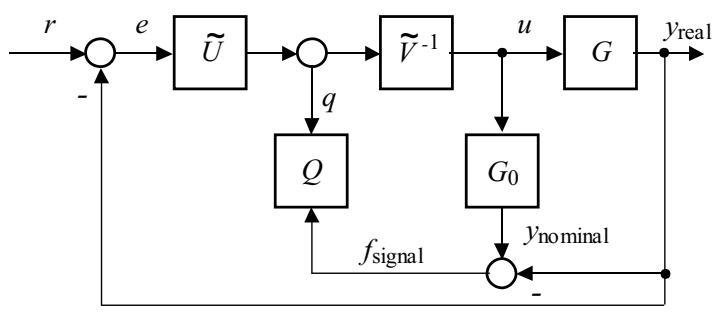

Fig. 3. GIMC structure

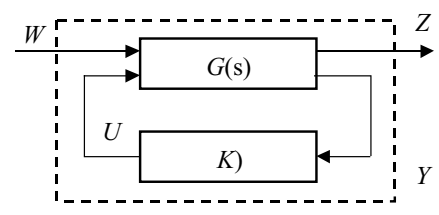

Fig. 4. $H_{\infty}$ robust control

\section{CONTROLLER DESIGN}

A high performance robust system can be designed in two steps:

1) Design $K_{0}=\tilde{V}^{-1} \tilde{U}$ to satisfy the system performance specifications with a nominal plant model $P_{0}$

2) Design $Q$ to satisfy the system robustness requirements. Note that the controller $Q$ will not affect the nominal performance of the system.

It should be emphasized that $K_{0}$ is not just any stabilizing controller as in most of controller parameterizations used in the literature, it is designed to satisfy certain performance specifications. For example, $K_{0}$ may be a simple proportional plus double-integral PI2 controller

$$
K_{0}=\frac{K_{p}(s+a)}{s^{2}}
$$

that satisfies our design specifications, in which case we can take $\tilde{U}=1$ and $\tilde{V}=K_{0}=\frac{K_{p}(s+a)}{s^{2}}$. The output error $f$ defined in Fig. 3 is the residual signal. In the fault diagnosis literature, $f$ is used to detect the possible faults in actuator and/or sensors. $f=0$ signifies nominal system without any failure. If $f=1$ a robust controller is implemented using the standard feedback structure shown in Fig. 4. The fault-tolerant controllers can be designed such that they provide adequate performance when there are no faults in the systems and as much tolerance as possible using a robust controller in the presence of the faults.

Such controllers can be designed in two steps:

1) Design $K_{0}=\tilde{V}^{-1} \tilde{U}$ to satisfy the performance for a nominal system.

2) Design $Q$ to tolerate possible actuators and/or sensors failures (and model uncertainties). This $Q$ can be found using standard robust control technique, fuzzy control, sliding mode control, etc. In our case, we use the robust $H_{\infty}$ technique.

\section{ROBUSTIFICATION}

In this section, the design of controller $K$ for a high degree of system robustness is considered.

Consider the system described by the block diagram of the Fig.figure6, where $G$ is the generalized plant and $K$ is the controller. Only finite-dimensional linear time invariant (LTI) systems and controllers will be considered in this paper. The generalized plant $G$ contains what is usually called the plant in a control problem including all the weighting functions. The signal $w$ contains all external inputs, including disturbances, sensor noise, and commands; the output $z$ is an error signal; $y$ is the measured variables; $u$ is the control input. The diagram is also referred to as a linear fractional transformation (LFT) on $K$, and $P$ is called the coefficient matrix for the LFT. The resulting closed-loop transfer function from $w$ to $z$ is denoted by $T_{z w}$. The problem of $H_{\infty}$ control is to synthesize a controller $K$ which stabilises the system $G$ and minimize the norm $H_{\infty}$ of $T_{z w}[19]$.

$$
G=\left[\begin{array}{ccc}
A & B_{1} & B_{2} \\
C_{1} & 0 & D_{12} \\
C_{2} & D_{21} & 0
\end{array}\right]
$$

The following assumptions are made:

1) $\left(A, B_{1}\right)$ is stabilizable and $\left(C_{1}, A\right)$ is detectable.

2) $\left(A, B_{2}\right)$ is stabilizable and $\left(C_{2}, A\right)$ is detectable.

3) $D_{12}^{\prime}\left[\begin{array}{ll}C_{1} & D_{12}\end{array}\right]=\left[\begin{array}{ll}0 & I\end{array}\right]$.

4) $\left[\begin{array}{ll}B_{1} & D_{21}\end{array}\right]^{T} D_{21}^{\prime}=\left[\begin{array}{ll}0 & I\end{array}\right]^{T}$.

The problem of $H_{\infty}$ control is to synthesize a controller $K$ which stabilises the system $G$ and minimizes the $H_{\infty}$ norm of $\left\|T_{z w}\right\|_{\infty}$.

Recall that the $H_{\infty}$ controller is

$$
K_{\infty}=\left[\begin{array}{cc}
\hat{A}_{\infty} & -Z_{\infty} L_{\infty} \\
F_{\infty} & 0
\end{array}\right],
$$




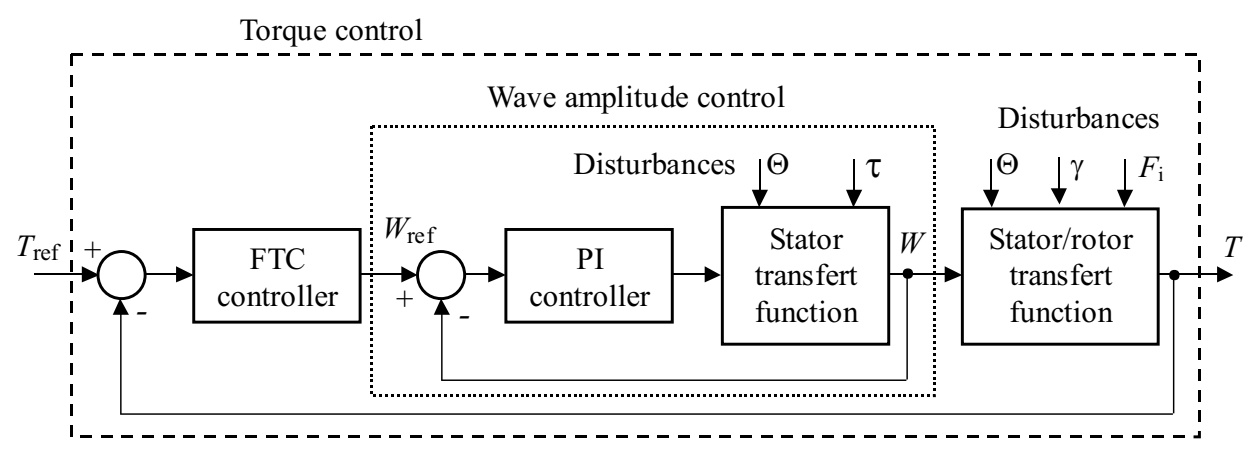

Fig. 5. Cascaded controllers with inner-loop control ( $P I$ controller of the wave amplitude control of the stator) and FTC controller for the torque control loop

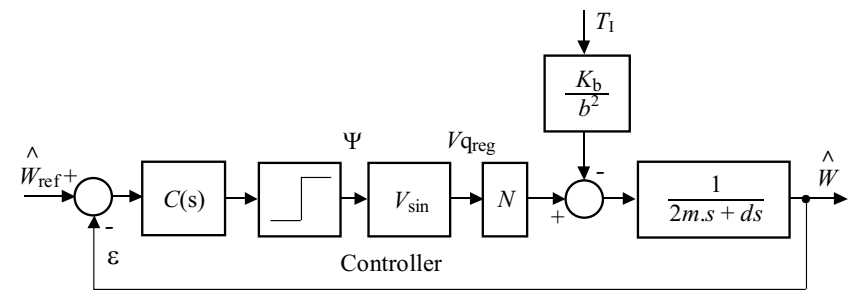

Fig. 6. Block diagram $H_{w}(s)$ of the wave amplitude control of the stator

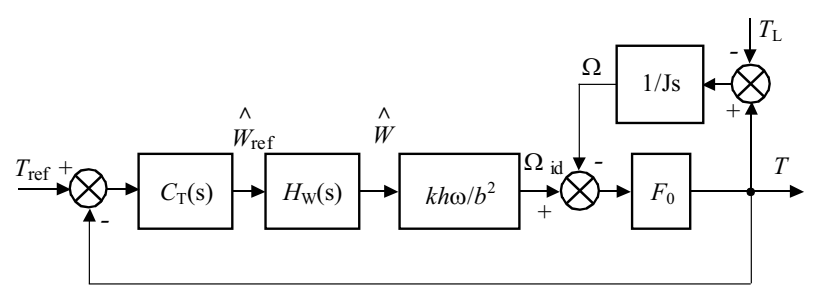

Fig. 7. Outer torque control loop

$$
\hat{A}=A+\gamma^{-2} B_{1} B_{1}^{\prime} X_{\infty}+B_{2} F_{\infty} C_{2}
$$

$F_{\infty}=-B_{2}^{\prime} X_{\infty}, L=-Y_{\infty} C_{2}, Z_{\infty}=\left(I-\gamma^{-2} Y_{\infty} X_{\infty}\right)^{-1}$

where $X_{\infty}=\operatorname{Ric}\left(H_{\infty}\right)$ and $Y_{\infty}=\operatorname{Ric}\left(J_{\infty}\right)$. The necessary and sufficient conditions for the existence of an admissible controller such that of $\left\|T_{z w}\right\|_{\infty}<\gamma$ are as follows [20]:

1) $H_{\infty} \in \operatorname{dom}($ Ric $)$ and $X_{\infty}=\operatorname{Ric}\left(H_{\infty}\right) \geq 0$.

2) $J_{\infty} \in \operatorname{dom}(\operatorname{Ric})$ and $X_{\infty}=\operatorname{Ric}\left(H_{\infty}\right) \geq 0$.

3) $\rho\left(X_{\infty} Y_{\infty}\right)<\gamma^{2}$.

where Ric stands for the standard solution of Ricatti equation.

The Hamiltonian matrices are defined as

$$
\begin{aligned}
H_{\infty} & =\left[\begin{array}{cc}
A & \gamma^{-2} B_{1} B_{1}^{\top}-B_{2} B_{2}^{\top} \\
-C_{1}^{\top} C_{1} & -A^{\top}
\end{array}\right] \\
J_{\infty} & =\left[\begin{array}{cc}
A^{\top} & \gamma^{-2} C_{1} C_{1}^{\top}-C_{2}^{\top} C_{2} \\
-B_{1} B_{1}^{T} & -A
\end{array}\right]
\end{aligned}
$$

\section{WAVE AMPLITUDE AND TORQUE CONTROLLER}

In this section, we present two cascaded controller architectures composed of both inner-loop and outer-loop controllers (Fig. 5). The inner loop controller regulates in $H_{w}(s)$ the vibrational travelling wave amplitude $\hat{W}$ provided by the stator with a simple $P I$ controller. The outer-loop controller consists of the torque feedback control of the motor shaft when subjected to variational loads with a simple FTC controller. In this study the disturbance of the outer loop is taken into account and the disturbance of the inner loop is neglected. Particularly, only an FTC controller for the torque control loop is proposed.

\subsection{Wave Amplitude Control}

The first block of the control scheme of the Fig. 6 regulates the wave amplitude. The transfer functions in the open-loop of the wave amplitude is given by [5]

$$
\widehat{W}=\frac{1}{w} \cdot \frac{N V_{q}-k \frac{h}{b^{2}} T_{i}}{\left(2 m+\left(k \frac{h}{b^{2}}\right)^{2} J\right) \cdot s+d_{s}} .
$$

For the synthesis of the wave amplitude controller $C(s), P I$ controller for $C(s)$ is chosen. Therefore, we let

$$
C(s)=K_{w} \frac{1+\tau_{w} s}{\tau_{w} s} .
$$

In order to limit the effect of the resonance, the time constant of the $P I$ controller is adjusted on its crest, so $\tau_{w}=1 /\left(2 \pi * F_{0}\right)$ with $F_{0}$ is the crest frequency. The pass-band of the open loop transfer function, which is tuned by $K_{w}$, is then adjusted so as to have a phase margin $\Delta \Phi=45$. For a pass-band of $3000 \mathrm{~Hz}$, we select $K_{w}=8 \times 10^{-3}$.

\subsection{Torque Control}

Piezoelectric actuators are often used to achieve position or active vibration control; in addition, they are well adapted for these applications. As for torque, or more 


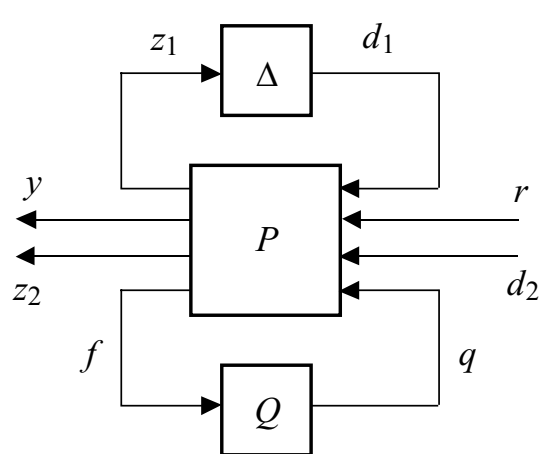

Fig. 8. The standard setup for design of $Q$ for systems with additive faults

generally force control, it is an important goal for haptic applications. For the actuators, this implies that control schemes are able to reproduce the force or the torque responses of virtual objects. Piezoactuators are also well suited to these tasks, given their generally low speed and capacity for producing significant force.

The block of the control scheme of the Fig. 7 regulates the torque. One of the drawbacks of this control is the acquisition of the actuator torque. Generally, for the torque sensor we use the strain gauge force. The transfer functions in the open loop of the torque is given by [5]

$$
T=H_{w}(s) k \frac{h}{b^{2}} w f_{0} \frac{\frac{J . s}{f_{0}}}{1+\frac{J . s}{f_{0}}} \widehat{W_{\text {ref }}} .
$$

The synthesis of the FTC torque controller $T_{L}(s)$ is obtained according to the implementation in Fig. 3, we
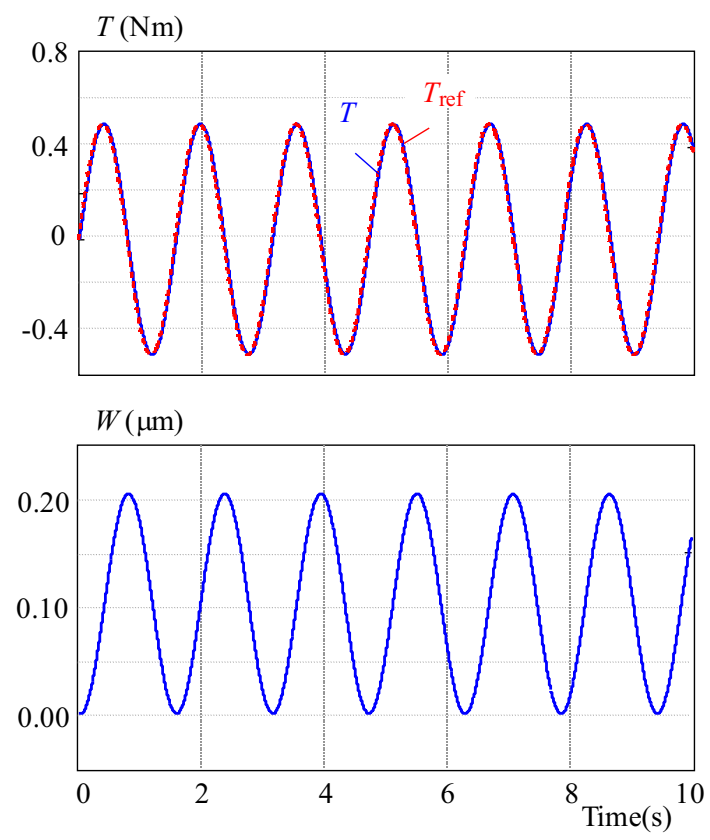

Fig. 9. Response of the USM motor without a fault sensor with PI2 controller use the proportional plus double-integral PI2 controller for the ideal model and the $H_{\infty}$ controller in the presence of the fault. For the ideal model, the loop controller is chosen so as to minimize static error. Thus, a corrector with a double integration is chosen, $T_{c}(p)=K_{c}((1+$ $\left.\left.\tau_{c} p\right) / \tau_{c} p^{2}\right), K_{c}, \tau_{c}$ are computed in order to ensure a good margin of stability. The $H_{\infty}$ controller $Q$ can be designed using the standard robust techniques as shown in Fig. 8, where $\Delta$ includes the additive fault.

The fault tolerant control problem depends strongly on the type of faults that can appear in the system. In this paper, the faults are described as additive faults. In connection with FTC, this might not be very useful. The reason is that the additive faults can be considered as external input signals to the system. which will not cause any changes in the system dynamics. Specifically, they are not able to change the stability of the closed-loop system but the performance of the system will be affected.

The synthesis of the $H_{\infty}$ torque controller $Q(s)$ is obtained according to the implementation in Fig. 8 and by using the command hinf syn of MATLAB $\mu$-Analysis and Synthesis Toolbox, the $H_{\infty}$ design of controller $Q(s)$ and weights may be carried out with reference to equation

$$
\begin{gathered}
z=\left[\begin{array}{c}
W_{1}(r-y) \\
W_{2} u
\end{array}\right], \quad w=\left[\begin{array}{c}
r \\
d_{1} \\
d_{2}
\end{array}\right] \\
y=\left[\begin{array}{c}
r+W_{3} d_{1} \\
y+W_{4} d_{2}
\end{array}\right], \quad u=u_{s}
\end{gathered}
$$
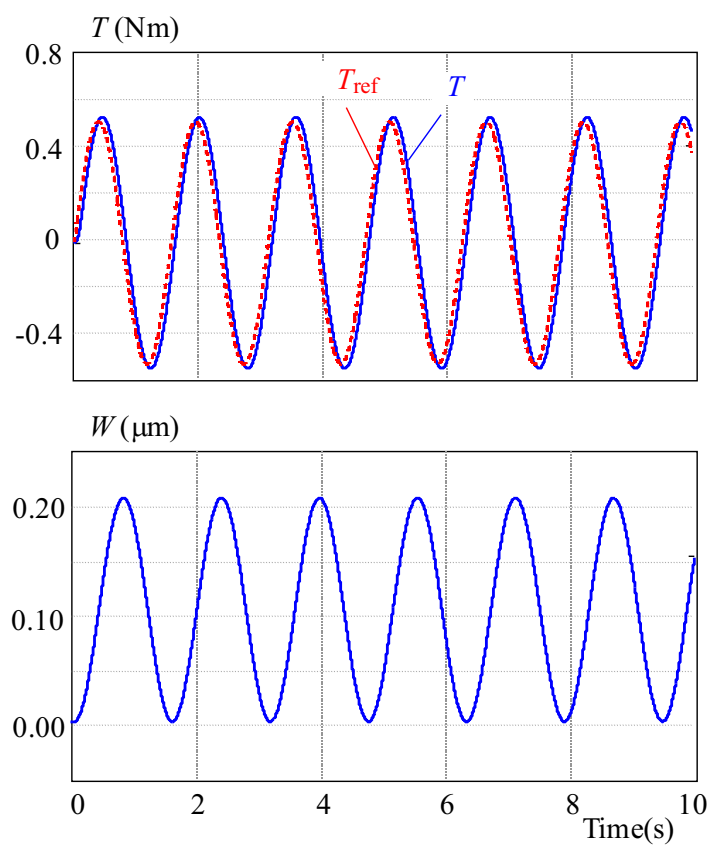

Fig. 10. Response of the USM motor without a fault sensor with $H_{\infty}$ controller 


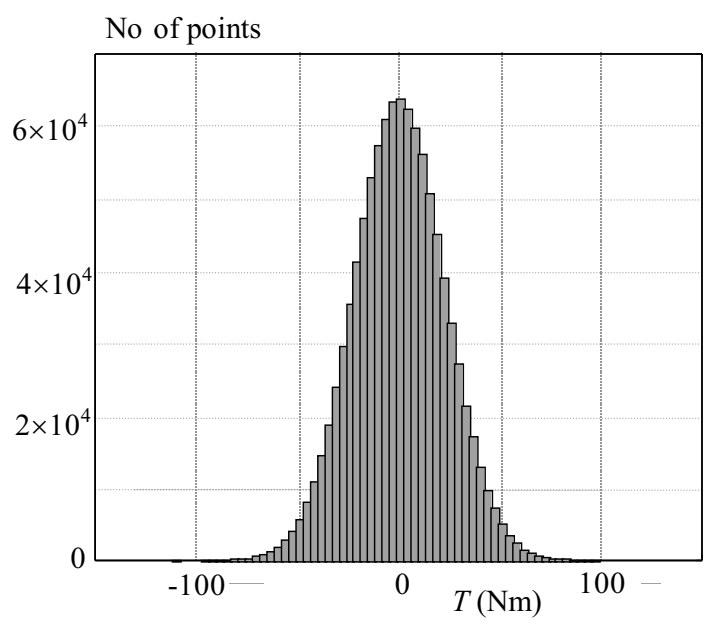

Fig. 11. Faulty sensor with histogram

\section{SIMULATION RESULTS}

In this part, an architecture for fault tolerant controller has been proposed, based on the GIMC structure shown in the block diagram in Fig. 3. There is a number of reasons for using the architecture from the Youla parametrization in connection with FTC. In this architecture, the $Q$ parameter represents the FTC characteristic of the controller. This means that the FTC part of the feedback controller is a modification of the existing controller. Thus, a controller change when a fault appears in the system is not a complete shift to another controller, but only a modification of the existing controller
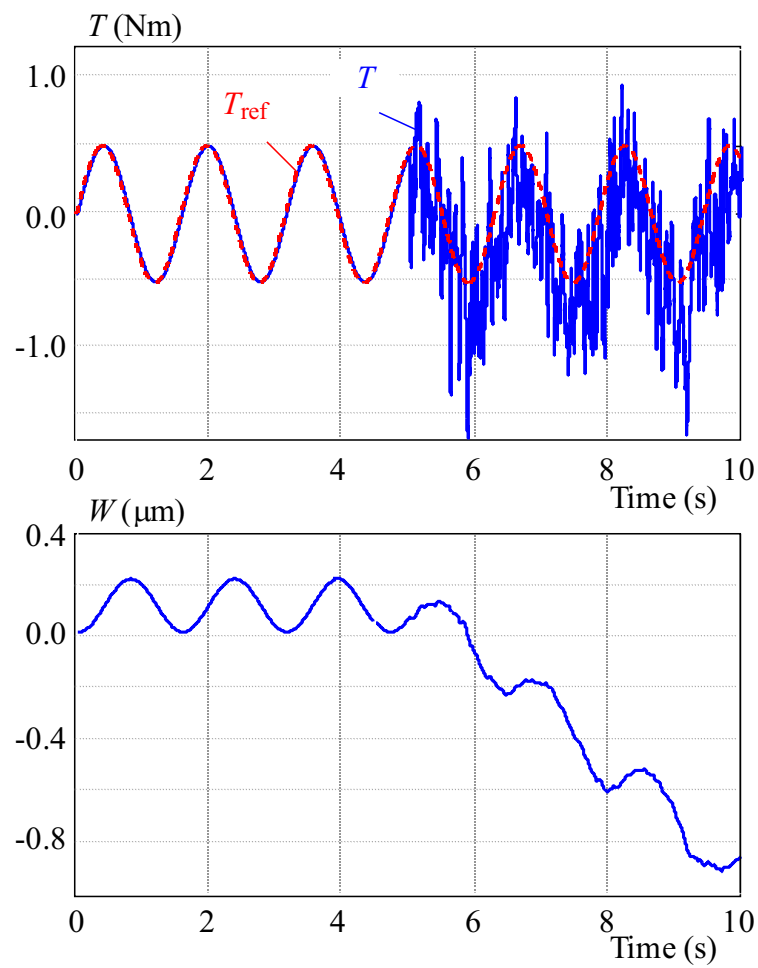

Fig. 12. Response of the USM motor with a fault sensor by adding a correction signal in the nominal controller, the $r$ signal in Fig. 3.

In short, for the torque control loop, we use the proportional plus double-integral PI2 controller for the ideal model and the $H_{\infty}$ controller in the presence of the fault. The simulation results given in Fig. 9 show the ideal torque and wave responses with a good performances of the proposed system using PI2 controller.

For the $H_{\infty}$ controller design, the specifications are taken to ensure the torque should track the reference torque. The control $U$ should not exceed a pre-specified saturation limit and rejects the fault. It ensures $\|f(G, Q)\|_{\infty}<\gamma$ for all $\|\Delta\|_{\infty}<1$. The controller synthesis is based on the equation (11) where the weighting matrices are chosen as follows [21]:

$w_{1}=\alpha_{1} \frac{10 s+10000}{s^{2}+10 s+0.1}, w_{2}=\alpha_{2} \frac{s}{s+1000}, w_{3}=\alpha_{3} \frac{s}{0.1 s+1}$, $w_{4}=\alpha_{4}$.

The weighting matrices for the design are used to scale these closed loop transfer functions to specify the frequency information on the tracking performances and on disturbance rejection. The weighting function $W_{1}$ of the error is chosen in such a way to make the norm from $r$ to $W_{1}(r-y)$ small leading to the choice of a transfer function of the form $W_{1}$. The choice of weighting function $W_{2}$ is based on limiting the saturation with the control $U$. Finally the choices of weighting functions $W_{3}$ and $W_{4}$ are made in order to have a loop gain large at low frequencies to achieve good performance and small at high frequencies to guard against unmodeled actuator dynamics saturation and noise.
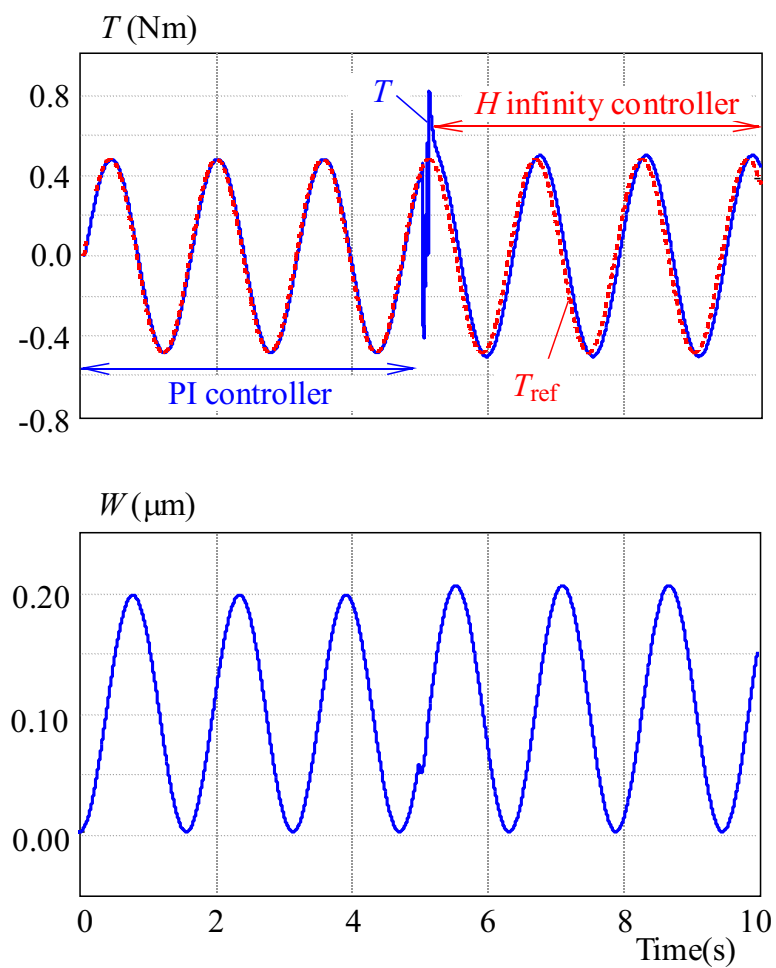

Fig. 13. Response of the USM motor using FTC controller at 5 Sec 

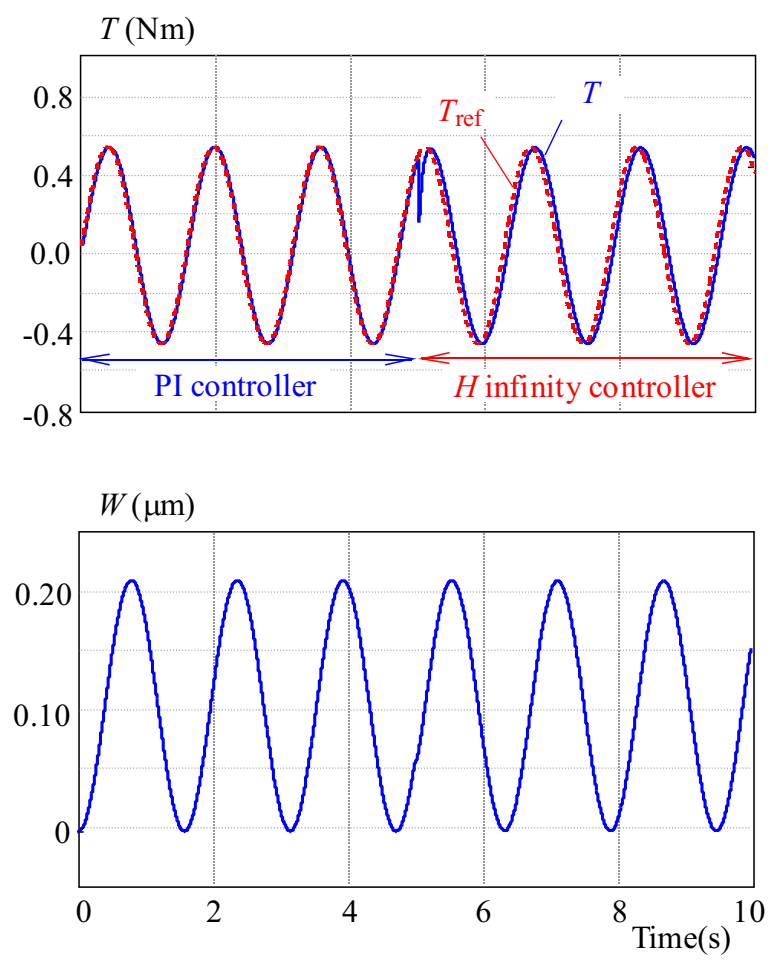

Fig. 14. Response of the USM motor using FTC controller at $5.1 \mathrm{Sec}$

As an illustration of the controller, the Fig. 10 shows the response of our system using $H_{\infty}$ controller with an acceptable performance.

\subsection{Simulation Results}

For FTC architecture, the system has been simulated with a sinusoidal reference and a sensor fault appears at $t=5 \mathrm{sec}$, the noisy sensing signals provided by the strain gauge force sensors is given as a normally Gaussian distributed random signal with 0.2 mean value and a variance at 250 as shown in Fig. 11. The results of the simulations are shown in Fig. 12, the proportional plus double-integral PI2 can not ensure good response in presence of the fault sensor.

A sinusoidal response of the system with fault induced by a noisy sensing torque signal applied on a USM motor (random disturbance) is shown in Fig. 12. It can be seen directly from this figure that the faulty closed-loop system is unstable. For stabilizing the faulty servo system a $Q$ controller needs to be included. As calculated above, the robust controller $Q$ can be applied for stabilizing the faulty closed-loop system. In this example, the $Q$ controller is implemented with a switching system which is activated by the residues signal $f$. If the system model with its parameters are not well identified, which is correct in real case, so the inner feedback $f$ will be already different to zero, In order to resolve this identification problem, we can impose the following condition to $f$.

$$
\begin{aligned}
& f=1 \quad \text { if } \quad T(P)-T\left(P_{0}\right)>T_{n} / 10, \\
& f=0 \quad \text { if } \quad T(P)-T\left(P_{0}<T_{n} / 10 .\right.
\end{aligned}
$$

$T(P), T\left(P_{0}\right)$ are respectively the torque of the real and nominal system. $T_{n}$ is the nominal torque given by the manufacturer.

Based on the design of $Q$ (see Fig. 8), the sinusoidal response of the USM motor is shown in Figs. 13 and 14 where it should be noticed that the standard robust controller is independent of the nominal PI2 controller. In the worst case, our controller implementation will be equivalent to the existing robust control design. Similar results of robustness and fault-tolerance will be obtained in the presence of the faults produced by temperature, mechanical stress and noisy sensing signals during mechatronics tasks (see Fig. 5).

Of course, if there is no uncertainty, proposed controller will perform as well as a nominal controller does. In fact, our framework provides a great flexibility in controller design, for example, one could still use all the robust and $H_{\infty}$ design techniques here. All one has to do is to start with a good performance controller and then follow the standard robust control design procedure to find the robust controller $Q$. The only difference is that one may not be interested in using $Q$ into the controller parametrization to find the total controller, rather one may implement the performance controller and the robust controller $Q$ separately.

\section{CONCLUSION}

In this paper, an architecture for fault tolerant control has been used for driving in closed-loop a piezoelectric travelling-wave ultrasonic motor. By applying the GIMC structure, an additional controller parameter has been introduced as the main tool to achieve fault tolerance. A feature of the GIMC structure is that it automatically includes a diagnostic signal. The presented simulation results show that the GIMC provides adequate performance when there are no faults in the system and provides tolerance by using a $H_{\infty}$ robust controller. In the future, the authors shall introduce this architecture fault tolerant control with the speed control and shall integrate the controller architecture into the ultrasonic motor for a fault-tolerant on-board mechatronics system.

\section{REFERENCES}

[1] BOUKHNIFER, M.-CHAIBET, A.: Fault Tolerant Control of Piezoelectric Traveling Wave Ultrasonic Motor, IEEE CCAConference on Control Applications, Saint Petersburg, Russia. , July 8-10, 2009.

[2] SHERRIT, S.et al : Resonance Analysis of High Temperature Piezoelectric Materials for Actuation and Sensing, SPIE Smart Structures Conference, vol. 5387-58, 2004.

[3] MORITA, T. et al : Rotational Feedthrough using an Ultrasonic Motor and its Performance in Ultra High Vacuum Conditions, Vacuum 70 (2003), 53-57.

[4] BAR-COHEN, Y.-BAO, X.-GRANDIA, W.: Rotary Ultrasonic Motors Actuated by Travelling Flexural Waves, SPIE 6th 
Annual International Symposium on Smart Structures and Materials, March 1999 .

[5] GIRAUD, F.-SEMAIL, B.-AUDREN, J.-T.: Analysis and Phase Control of a Piezoelectric Travelling-Wave Ultrasonic Motor for Haptic Stick Application, IEEE Transactions On Industry Applications 40 No. 6 ().

MAAS, J.-SCHULTE, T.-FRÖHLEKE, N.: Model-Based Control for Ultrasonic Motors, IEEE/ASME Transaction on Mechatronics 5 No. 2 (June 2000).

[7] PETIT, L.et al: Frequency behaviour and Speed Control of Piezomotorsjour Sensor and Actuator.

[8] MAAS, J.-IDE, P.—FRÖHLEKE, N.—GROTSTOLLEN, H. : Simulation Model for Ultrasonic Motors powered by Resonant Converters, Industry Application Conference 1995, IAS'95, vol. 1 , pp. 111-120 .

MAAS, J.-SCHULTE, T.: High Performance Speed Control for Ultrasonic Motors, IEEE/ASME International Conference on Advanced Intelligent Mechatronics, 1999.

GOUATARBES, J.-BOUKHNIFER, M.-FERREIRA, A.AUBRY, D.-MAGNAN, P.: Robust Control for Ultrasonic Motor Operating within Harsh Environments, IEEE/ASME International Conference on Advanced Intelligent Mechatronics, 2007

[11] SERON, M.-ROMERO, M. E.-De DONA, J. A.: Sensor Fault Tolerant Control of Induction Motors, International Federation of Automatic Control World Conference, July 6-11, 2008

[12] LIAW, H. C.-SHIRINZADEH, P.-SMITH, J. : International Federation of Automatic Control World Conference, July 6-11, 2008 .

[13] OUDGHIRI-BENTAIE, M.-CHADLI, M.-El HAJJAJI, A. : Control and Sensor Fault-Tolerance of Vehicle Lateral Dynamicsinbook International Federation of Automatic Control World Conference.

[14] BAL, G.: A Digitally Controlled Drive System for Travelling-wave Ultrasonic Motor, Turk J. Elec, Engin. 11, 155-168
[15] CHEN, J. S.-LIN, I. D.: Toward the Implementation of an Ultrasonic Motor Servo Drive using FPGA, Mechatronics 12 (2002), 511-524 .

[16] ZHOU, K.-REN, Z.: A New Controller Architecture for High Performance, Robust, and Fault-Tolerant Control, IEEE Transactions on Automatic Control 46 No. 10 (Oct 2001 ).

[17] STOUSTRUP, J.-NIEMANN, H.: Fault Tolerant Feedback Control using the Youla Parameterization, Proceedings of the $6^{\text {th }}$ European Control Conference, Porto, Portugal, Sept 2001, pp. 1970-1974 .

[18] MORARI, M.-ZAFIRIOU, E. : Robust Process Control, Prentice-Hall, Upper Saddle River, NJ , 1989.

[19] DOYLE, J.-GLOVER, K.-KHARGONEKAR, P.-FRANCIS, B.: State-Space Solutions to Standard $\mathrm{H}_{2}$ and $H_{\infty}$ Control Problems, IEEE Transactions on Automatic Control AC-34 No. 8, (Aug 1989 ), 831-847.

[20] ZHOU, K.-DOYLE, J.-GLOVER, K. : Robust and Optimal Control, Prentice Hall, 1995.

[21] LUndstrom, P.-SKOGSTAD, S.-WANG, Z. C.: Weight Selection for $H_{\infty}$ and $\mu$-Control Methods: Insights and Examples from Process Control, Workshop on $H_{\infty}$ control, Brighton, UK, pp. 139-1991 .

Received 29 September 2011

Moussa Boukhnifer is an associate professor at Laboratoire Commande et Systèmes, ESTACA of Paris (France). He received the MSc degree in electrical engineering from INSA of Lyon, France, in 2002. In December 2005, he received the doctorate degree in Control and Engineering from the University of Orléans, France. In 2007, he was a Researcher at INRETS of Paris. His main research interests are focused on the design, modeling and fault tolerant control with its applications to power electronics and electrical drives.

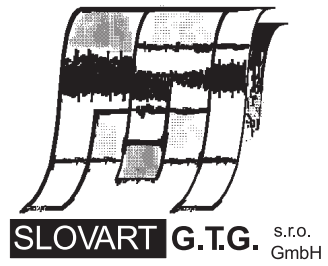

EXPORT - IMPORT
EXPORT - IMPORT

of periodicals and of non-periodically printed matters, books and CD-ROMs

Krupinská 4 PO BOX 152, 85299 Bratislava 5, Slovakia tel: ++421 263839 472-3, fax: ++421 263839485 info@slovart-gtg.sk; http://www.slovart-gtg.sk

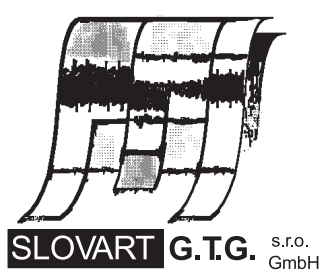

EXPORT - IMPORT 